\title{
Endocytosed nanogold fiducials for improved in-situ cryo-electron tomography tilt-series alignment
}

Citation for published version (APA):

Berger, C., Ravelli, R. B. G., Lopez-Iglesias, C., \& Peters, P. J. (2021). Endocytosed nanogold fiducials for improved in-situ cryo-electron tomography tilt-series alignment. Journal of Structural Biology, 213(1), [107698]. https://doi.org/10.1016/j.jsb.2021.107698

Document status and date:

Published: 01/03/2021

DOI:

10.1016/j.jsb.2021.107698

Document Version:

Publisher's PDF, also known as Version of record

Document license:

Taverne

Please check the document version of this publication:

- A submitted manuscript is the version of the article upon submission and before peer-review. There can be important differences between the submitted version and the official published version of record.

People interested in the research are advised to contact the author for the final version of the publication, or visit the DOI to the publisher's website.

- The final author version and the galley proof are versions of the publication after peer review.

- The final published version features the final layout of the paper including the volume, issue and page numbers.

Link to publication

\footnotetext{
General rights rights.

- You may freely distribute the URL identifying the publication in the public portal. please follow below link for the End User Agreement:

www.umlib.nl/taverne-license

Take down policy

If you believe that this document breaches copyright please contact us at:

repository@maastrichtuniversity.nl

providing details and we will investigate your claim.
}

Copyright and moral rights for the publications made accessible in the public portal are retained by the authors and/or other copyright owners and it is a condition of accessing publications that users recognise and abide by the legal requirements associated with these

- Users may download and print one copy of any publication from the public portal for the purpose of private study or research.

- You may not further distribute the material or use it for any profit-making activity or commercial gain

If the publication is distributed under the terms of Article $25 \mathrm{fa}$ of the Dutch Copyright Act, indicated by the "Taverne" license above, 
Short Communication

\title{
Endocytosed nanogold fiducials for improved in-situ cryo-electron tomography tilt-series alignment
}

\author{
Casper Berger, Raimond B.G. Ravelli, Carmen López-Iglesias, Peter J. Peters \\ Division of Nanoscopy, Maastricht Multimodal Molecular Imaging Institute, Maastricht University, the Netherlands
}

\section{A R T I C L E I N F O}

\section{Keywords:}

Cryo-electron tomography

Cryo-electron microscopy

Cryo-focused-ion beam lamella

Tilt-series alignment

Nanogold fiducials

Bovine serum albumin bsa-gold

\begin{abstract}
A B S T R A C T
Cryo-electron tomography (CET) on cryo-focused ion beam (FIB)-milled lamellae is becoming a powerful technique for determining the structure of macromolecular complexes in their native cellular environment. Prior to tomogram reconstruction, CET tilt-series recorded on FIB lamellae need to be aligned. Traditionally, CET tiltseries alignment is performed with 5-20 nm gold fiducials, but it has thus far proven difficult to apply this to FIB lamellae of eukaryotic cells. In here, we describe a simple method to allow uptake of bovine serum albumin (BSA)-gold fiducials into mammalian cells via endocytosis, which can subsequently be used as fiducials for tiltseries alignment of cryo-FIB lamellae. We compare the alignment of tilt-series with BSA-gold fiducials to fiducialless patch-tracking, and find better alignment results with BSA-gold. This technique can contribute to understand cells at a structural and ultrastructural level with both cryo- and room-temperature electron tomography. Furthermore, fluorescently labeled BSA-gold has the potential to be used as fiducials for correlative light and electron microscopy studies.
\end{abstract}

\section{Introduction}

Recent advances in cryo-electron microscopy have established single-particle analysis and cryo-electron tomography (CET) combined with subtomogram averaging as powerful methods for determining the structure of proteins (Kuhlbrandt, 2014; Turoňová et al., 2017; O'Reilly et al., 2020). Where single particle relies on the biochemical isolation of proteins, CET allows proteins to be studied in the native context of the cell. However, the increased probability of inelastic scattering events of electrons with increased sample thickness makes it impossible to use CET to image most areas of mammalian cells. Where sample thinning with vitreous cryo-sectioning causes artifacts such as compression and crevasses (Pierson et al., 2011), it has recently become possible to overcome these limitations by using a cryo-focused ion-beam (FIB) to prepare $\sim 200 \mathrm{~nm}$-thick lamellae of cells (Marko et al., 2007; Mahamid et al., 2016; Schaffer et al., 2017). This allows for the structural study of protein complexes in their native cellular environment (Weiss et al., 2019; Albert et al., 2020; Wolff et al., 2020).

In order to accurately reconstruct a 3-dimensional tomographic volume from a CET tilt-series, the individual tilted images need be aligned to correct for sample and stage movements, after alignment of different frames recorded at the same tilt angle (Zheng et al., 2017). The generally preferred method is to add 5-20 nm gold nanoparticles to the sample prior to vitrification to act as fiducials during tilt-series alignment (Gruska et al., 2008; Dobro et al., 2017). The use of fiducials allows for accurate tilt-series alignment by correcting for non-rigid registration due to beam and stage-induced shifts as well as sample alterations (Karimi Nejadasl et al., 2013; Mastronarde and Held, 2017; Fernandez et al., 2018, 2019). However, adding fiducials to FIB-milled lamellae of eukaryotic cells has so far proven to be difficult. Nanogold particles in 2methylpentane can be used to deposit fiducials to the lamella surface post-milling (Harapin et al., 2015), but so far successful cases employing this method are limited. Fiducials can also be deposited on the grid before vitrification and used in FIB-wedges, because the grid foil is still present below the thinned cellular material (Jasnin et al., 2019). However, they will not be visible in FIB-lamellae, where also the material below the lamella is removed, which causes the grid foil (with the gold fiducials) to only intersect with a small section of the tilted lamella.

Many fiducial-less alignment methods that use cross-correlations or feature tracking to create virtual fiducials have been developed (Owen and Landis, 1996; Lowe, 2004; Bay et al., 2008; Han et al., 2014; Noble and Stagg, 2015; Chen et al., 2019), which are generally used in CET studies on FIB-lamellae (Mahamid et al., 2016; Bäuerlein et al., 2017; Albert et al., 2020). However, fiducial-less alignment can be challenging

\footnotetext{
* Corresponding author.

E-mail address: pj.peters@maastrichtuniversity.nl (P.J. Peters).
} 

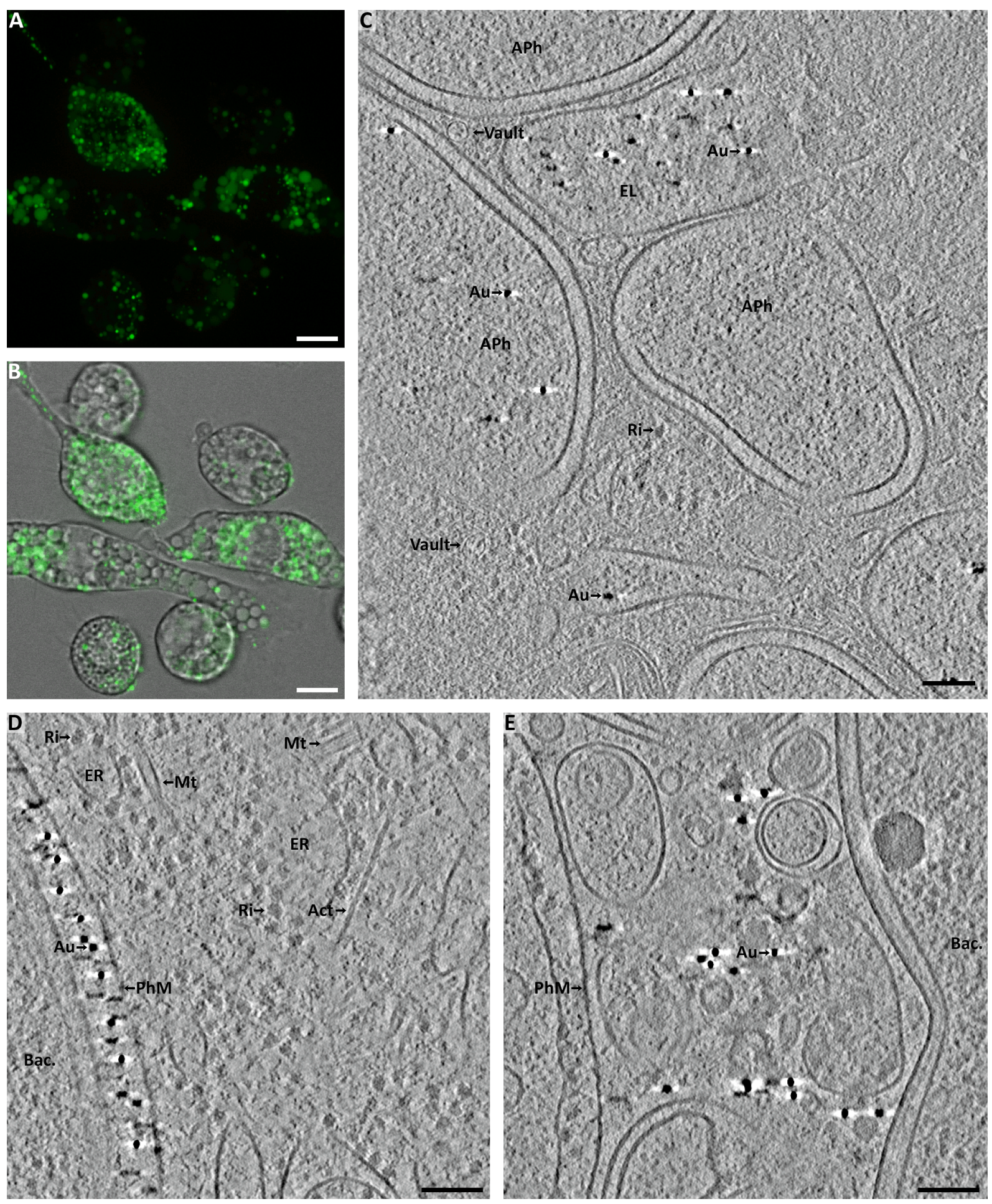

Fig. 1. The use of BSA-gold fiducials to align CET tilt-series recorded on FIB-lamellae. (A) Distribution of $5 \mathrm{~nm}$ BSA-gold labeled with Alexa 488 in J774 mouse macrophages after $90 \mathrm{~min}$ of incubation. Scale bar: $10 \mu \mathrm{m}$. (B) Fluorescence image of A overlaid with transmitted light imaging of the cells. (C) Tomographic slice of a dendritic cells differentiated from primary human blood monocytes infected with M. marinum and incubated for 60 min prior to vitrification with $10 \mathrm{~nm}$ BSA-gold. The tomogram was recorded on an area where no intracellular bacteria are present. BSA-gold is present in different endo-lysosomal organelles (EL), including double membrane-enclosed organelles of autophagosomal origin (APh), which may contain BSA-gold because they have fused with endosomal organelles. A total of 70 BSAgold particles (Au) are present in this tomogram. Many cytosolic ribosomes (Ri) and two vault complexes (Vault) (Woodward et al., 2015) can be observed in this slice as well. Scale bar: $100 \mathrm{~nm}$. (D) Tomographic slice of primary human blood monocytes differentiated into dendritic cells and infected with Y. enterocolitica. A large number of $10 \mathrm{~nm}$ BSA-gold particles (Au) are surrounding the bacterium in the tomographic volume because $Y$. enterocolitica is covered in a dense coat of the bacterial adhesin YadA (Hoiczyk, 2000), which binds to the BSA-gold. A total of 109 BSA-gold particles are present in this tomogram. The bacterium (Bac.) is enclosed by the phagosomal membrane (PhM). In the cytosol, the endoplasmic reticulum (ER) can be observed surrounded by ribosomes (Ri), actin (Act), and microtubules (Mt). Scale bar: $100 \mathrm{~nm}$. (E) Tomographic slice of the mouse macrophage cell line J774 with intracellular E. coli. bacteria (Bac.) after phagocytosis. The bacteria are surrounded by a phagosomal membrane (PhM). The cellular material present in the organelle is likely from phagocytosis of cellular debris from apoptotic cell. A total of 127 BSA-gold particles (Au) are present in this tomogram. Scale bar: $100 \mathrm{~nm}$. 
with the low signal-to-noise ratio typically present in CET tilt-series, in particular at high tilt angles. In situ structural research with CET on FIBlamellae would benefit from a simple method to add nanogold fiducials to FIB-lamellae.

In this technical note, we describe how endocytosed, $10 \mathrm{~nm}$ bovine serum albumin (BSA)-coated gold nanoparticles can be used as fiducial for tilt-series alignment of cryo-tomograms recorded on FIB-lamellae of mammalian cells and show that the use of BSA-gold for tilt-series alignment results in higher quality tomographic reconstructions compared to fiducial-less alignment. This method may be used to better study the structural states of protein complexes in the native context of the cell with CET on FIB-lamellae and offers the potential to be used as fiducials for correlative light and electron microscopy as well.

\section{Sample preparation}

\subsection{Cell culture \& uptake of BSA-gold}

Primary blood monocytes were isolated from a human blood buffy coat (Sanquin) with RosetteSep (Stemcell Technologies) as described by the manufacturer. Isolated monocytes were kept at $37{ }^{\circ} \mathrm{C}$ and $5 \% \mathrm{CO}_{2}$ in AIM V medium (Thermo Fisher Scientific, cat. 12055083) in non-tissue culture-treated petri dishes (Greiner, ref 633179) and differentiated into dendritic cells with $25 \mathrm{ng} / \mathrm{ml}$ interleukin-4 (IL-4) (PeproTech, cat 20004) and $200 \mathrm{ng} / \mathrm{ml}$ granulocyte-macrophage colony-stimulating factor (GM-CSF) (PeproTech, cat 300-03) for 6 days. J774 cells were grown in RPMI (Thermo Fisher Scientific. Cat. 11875093) supplemented with $10 \%$ fetal bovine serum.

For vitrification, 400.000 cells were transferred to a $35 \mathrm{~mm}$ petri dish (Greiner,ref 627160) containing 8 UltrAufoil 200-mesh R2/2 grids (Russo and Passmore, 2014). Cells were infected with either Yersinia enterocolitica AD4334 (E40 $\Delta$ blaA $\Delta$ asd $\Delta$ yopH $\Delta$ yopO $\Delta$ yopP $\Delta$ yopE $\Delta$ yopM $\Delta$ yopT) SctV-mCherry or Escherichia coli Rosetta 2(DE3) expressing the plasmid pQLinkN:EccB1-EccCa-EccCb1-mNG-His6EccD1-EccE1 for $1 \mathrm{~h}$ before vitrification or infected with Mycobacterium marinum $\mathrm{M}^{\mathrm{USA}} 16 \mathrm{~h}$ before vitrification. At $1 \mathrm{~h}$ before vitrification, $5 \mu \mathrm{l}$ of $10 \mathrm{~nm}$ BSA-gold fiducials with an optical density $\left(\mathrm{OD}_{600}\right)$ of 235 (Cell Microscopy Core, University Medical Center Utrecht) were added to the cells in a total volume of $1 \mathrm{ml}$ of medium with a final $\mathrm{OD}_{600}$ of 1.2 and allowed to be taken up via endocytosis.

For visualizing the distribution of BSA-gold with fluorescence microscopy, J774 mouse macrophages in RPMI medium were allowed to take up $5 \mathrm{~nm}$ BSA-gold fluorescently labeled with Alexa Fluor 488 dye (Cell Microscopy Core, University Medical Center Utrecht) with a final $\mathrm{OD}_{600}$ of 2.5 for $90 \mathrm{~min}$. For visualizing the distribution of BSA-gold with EM on epon sections, J774 mouse macrophages in RPMI medium were allowed to take up $10 \mathrm{~nm}$ BSA-gold with a final $\mathrm{OD}_{600}$ of 1.2 for $60 \mathrm{~min}$.

\subsection{Fluorescence microscopy}

Live $\mathrm{J774}$ cells were imaged at $37{ }^{\circ} \mathrm{C}$ by recording Z-stacks with a CorrSight spinning disk confocal fluorescence microscope (Thermo Fisher Scientific) with a $40 \times$ air objective (Zeiss, 420363-9901-000) with $488 \mathrm{~nm}$ excitation in spinning disk mode. For visualization in Fig. $1 \mathrm{~A}$ and $\mathrm{B}$, maximum intensity projections of the Z-stacks were overlaid with in-focus transmitted light images of the cells. For Supplementary Movie 1, individual optical slices are shown as a movie.

\subsection{Epon sectioning, EM overview acquisition and gold quantification}

After uptake of $10 \mathrm{~nm}$ BSA gold, J774 cells were chemically fixed with $2.5 \%$ glutaraldehyde in $0.1 \mathrm{M}$ phosphate buffer for $60 \mathrm{~min}$ at room temperature. Fixed cells were washed twice in $0.1 \mathrm{M}$ phosphate buffer, followed by washing twice in $0.1 \mathrm{M}$ cacodylate buffer. Postfixation was performed for $1 \mathrm{~h}$ at $4{ }^{\circ} \mathrm{C}$ in $0.1 \mathrm{M}$ cacodylate buffer with $1 \%$ osmium tetroxide and $1.5 \% \mathrm{~K}_{3} \mathrm{Fe}(\mathrm{CN})_{8}$, followed by washing 5 times in milli-Q.
Dehydration was performed in ethanol at room temperature and cells were embedded in Epon. 130-140 nm thin sections were prepared using a microtome.

EM overviews were acquired with a Tecnai Arctica (Thermo Fisher Scientific) equipped with a Falcon III direct electron detector (Thermo Fisher Scientific), operating at $200 \mathrm{kV}$. Tilescan overviews were acquired with MAPS 3.7 (Thermo Fisher Scientific) with a pixelsize of 9.8 $\AA$ and $1.5 \mu \mathrm{m}$ underfocus. Tilescan overviews were stitched with the FIJI grid/collection stitching plugin (Schindelin et al., 2012). Distribution of $10 \mathrm{~nm}$ BSA-gold within the cell was determined on the stitched overviews by picking BSA-gold particles with the IMOD function imodfindbeads (Mastronarde and Held, 2017), followed by manual evaluation and correction of the model where required. Distribution of BSA-gold was visualized using IMOD.

\subsection{Vitrification \& lamellae preparation}

Electron microscopy (EM) grids were vitrified with a modified vitrobot (Frederik and Hubert, 2005) (Thermo Fisher Scientific) with a custom-built jet vitrification device (Ravelli et al., 2020) and a force sensor feedback loop to accurately apply a specific blot force. On the sample side of the blotting pads, a sheet of Teflon was used and on the backside, Whatman filter paper (Sigma-Aldrich, cat. no. 1001-055). EM grids were blotted for $5 \mathrm{~s}$ with a $2 \mathrm{~s}$ wait time before plunging, a chamber humidity of $80 \%$ at $37{ }^{\circ} \mathrm{C}$ and a blot force of $2.45 \mathrm{~N}$.

Cryo-FIB lamellae were fabricated with a SCIOS FIB/SEM dual-beam (Thermo Fisher Scientific) which was upgraded to similar specifications as an Aquilos (Thermo Fisher Scientific). A thin ( $\sim 5-10 \mathrm{~nm}$, estimated based on the sputter-coating parameters) platinum layer was sputtercoated on the grid ( $6 \mathrm{~s}, 10 \mathrm{~W}, 600 \mathrm{~V}, 30 \mathrm{~mA})$ and an overview of the grid was acquired with the SEM in MAPS software 3.1 (Thermo Fisher Scientific). Sites for lamellae fabrication were chosen and the eucentric positions were determined prior to applying an organometallic platinum layer with the gas injection system at three different areas of the grid for $3 \mathrm{~s}$, resulting in an even layer thickness of approximately $1 \mu \mathrm{m}$ as observed with the TEM during FIB-lamella fabrication. Cryo-FIB lamellae were prepared with rectangular pattern milling or wedge premilling with a current between 0.05 and $1 \mathrm{nA}$ at an angle of $11^{\circ}$ relative to the grid with an initial width between 8 and $20 \mu \mathrm{m}$. A thin $(\sim 5$ $\mathrm{nm}$, as measured in the reconstructed tomograms) platinum layer was applied to the finished lamella $(3 \mathrm{~s}, 10 \mathrm{~W}, 600 \mathrm{~V}, 30 \mathrm{~mA})$.

\subsection{CET and tilt-series alignment}

CET was performed with a Tecnai Arctica (Thermo Fisher Scientific) equipped with a Falcon III direct electron detector (Thermo Fisher Scientific), with a pixel size of $2.73 \AA$ and operating at $200 \mathrm{kV}$ or a Titan Krios equipped with a $\mathrm{K} 2$ direct electron detector (Gatan), with a pixel size of $4.24 \AA$, operating at $300 \mathrm{kV}$ and equipped with a Gatan Imaging Filter operating with a slit width of $20 \mathrm{eV}$. On both electron microscopes, cameras were operated in electron counting mode. Low-dose overviews of the lamellae were acquired to record tilt-series of biological targets of interest. For the Tecnai Arctica, low-dose tilescan overviews were acquired with MAPS 3.7 (Thermo Fisher Scientific) with a pixelsize of 12.9 $\AA$, and $200 \mu \mathrm{m}$ underfocus. For the Titan Krios, single image low-dose overviews were acquired at $1200 \times$ magnification to capture the entire lamella in one image with $500 \mu \mathrm{m}$ underfocus. Tilt-series were acquired with a bidirectional tilt scheme with $3^{\circ}$ increments and an angular range of $51^{\circ}$ to $-51^{\circ}$ with correction for the pre-tilt of the lamella for the data acquired with a Tecnai Arctica or $2^{\circ}$ degree increments with an angular range of $50^{\circ}$ to $-50^{\circ}$ corrected for the pre-tilt of the lamella for the data acquired with a Titan Krios. Tilt-series were recorded with Tomography 4 software (Thermo Fisher Scientific), a total fluence of $100 \mathrm{e}-/ \AA^{2}$ and 4-6 $\mu \mathrm{m}$ underfocus.

Dose fractions were aligned with MotionCor2 version 1.1 (Zheng et al., 2017) and tilt-series were aligned with Etomo in IMOD version 

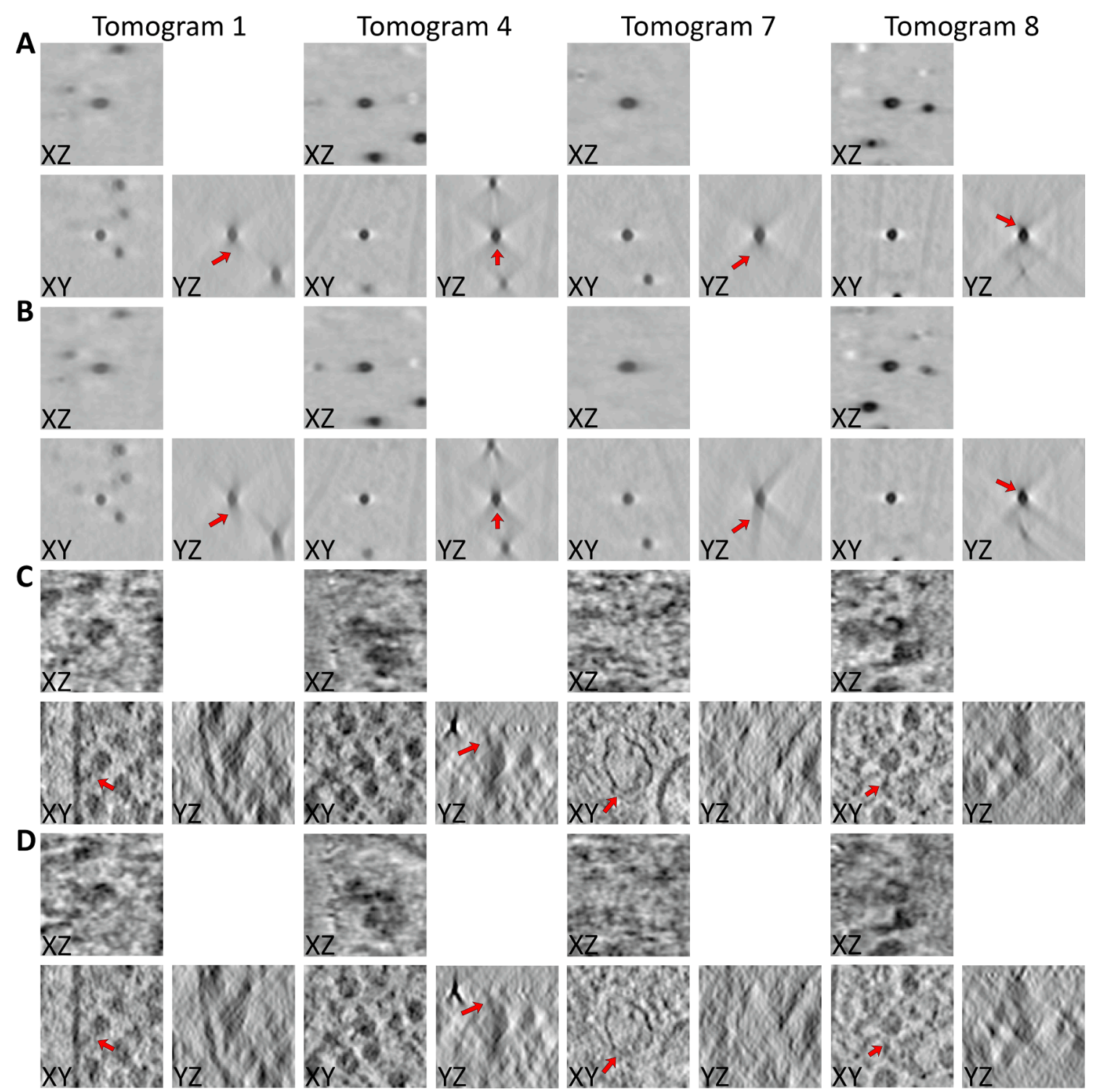

Fig. 2. Comparison of tilt-series alignment quality with fiducials compared to fiducial-less alignment. Tilt-series from several different lamellae were independently aligned with $10 \mathrm{~nm}$ BSA-gold as fiducials and fiducial-less patch-tracking. (A, B) XY, YZ and XZ slices of 4 different BSA-gold particles from different tomograms aligned with the fiducials (A) or aligned with fiducial-less patch-tracking (B). Red arrows indicate areas of particular difference. (C, D) XY, YZ and XZ slices of biological features from tomograms aligned with BSA-gold fiducials (C) or fiducial-less patch-tracking (D). Tomogram 1, 4, and 8 show bacterial ribosomes of $Y$. enterocolitica and tomogram 7 a vault complex of the infected host cell. Red arrows indicate areas of particular difference.

4.10.28 (Mastronarde and Held, 2017). For fiducial-based alignment, unbinned tilt-series were used to automatically create the initial seed model with up to 250 points, followed by automatic tracking. The resulting fiducial model was manually corrected for errors, missing points and more gold particles were added. For points with a high displacement compared to the fit determined on all the points (high residual error), the centering of the points on the fiducials was manually iteratively improved. For fiducial-less alignment, patch-tracking in Etomo was used on $4 \times$ binned tilt-series with a patch size of $200 \times 200$ pixels, a fractional overlap of 0.4 and 48 pixels away from the edges, resulting in $7 \times 7$ patches. Patches that were not tracking correctly were manually removed and tilt-images where most or all patches were not tracking correctly were excluded from being used to reconstruct the tomogram.

For comparing the alignment between fiducial-based and fiducialless alignment, tilt-series were independently reconstructed by weighted back projection for both methods, and alignment parameters were extracted from the project folders with a bash script. Values for the residual error were converted to unbinned pixels. For visual comparison between fiducial-based and fiducial-less alignment, 2D slices in $\mathrm{XY}, \mathrm{YZ}$ and XZ direction of the same BSA-gold or biological structures were extracted from both tomograms with a bash script. For visualization, reconstructed tomograms were filtered with TomDeconv (https://gith ub.com/dtegunov/tom_deconv/) with a signal-to-noise ratio falloff of 0.9 and the deconvolution strength set to 0.7. Movies of tomograms were recorded in IMOD.

\section{Nanogold in mammalian cells}

BSA-gold is generally not toxic to the cells and has been extensively used to study endocytosis and as therapeutic agents (Bolaños et al., 2019). BSA-gold is known to be endocytosed by mammalian cells within minutes into early endosomes, reaching late endosomal organelles within $1 \mathrm{~h}$ (Kleijmeer et al., 1997). We used fluorescently labeled, $5 \mathrm{~nm}$ BSA-gold to visualize its cellular distribution after endocytosis (Fig. 1A, B, Movie S1). 
Table 1

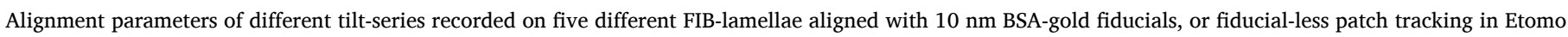

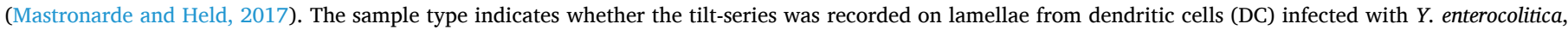

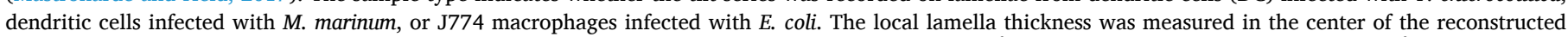

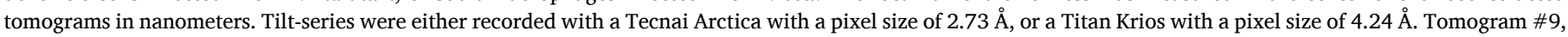

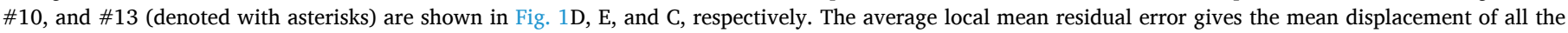

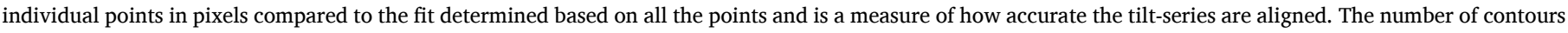

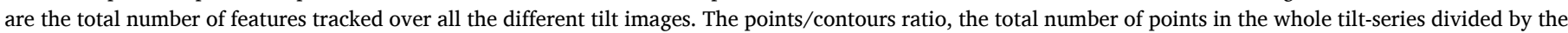

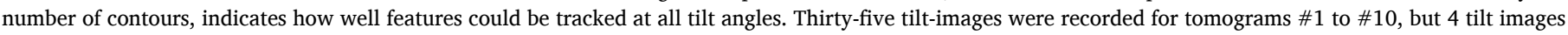

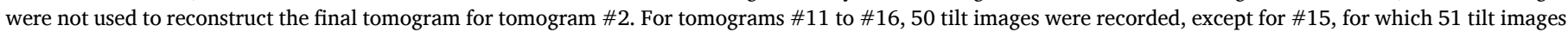
were recorded.

\begin{tabular}{|c|c|c|c|c|c|c|c|c|c|}
\hline \multirow[b]{2}{*}{ Tomogram } & \multirow[b]{2}{*}{ Sample type } & \multirow[b]{2}{*}{ Pixel size } & \multirow[b]{2}{*}{ Local lamella thickness (nm) } & \multicolumn{2}{|c|}{ Average local mean residual error } & \multicolumn{2}{|c|}{ Number of tracked contours } & \multicolumn{2}{|c|}{ Points/contours ratio } \\
\hline & & & & Gold fiducial & Fiducial-less & Gold fiducial & Fiducial-less & Gold fiducial & Fiducial-less \\
\hline$\# 1$ & DC - Y. ent. & 2.73 & 201 & 0.70 & 1.50 & 172 & 44 & 35.0 & 33.9 \\
\hline \#2 & DC - Y. ent. & 2.73 & 232 & 0.70 & 3.22 & 99 & 45 & 31.0 & 31.0 \\
\hline$\# 3$ & $D C-Y$. ent. & 2.73 & 148 & 1.06 & 0.98 & 91 & 45 & 35.0 & 33.2 \\
\hline$\# 4$ & $D C-Y$. ent. & 2.73 & 94 & 0.92 & 0.86 & 116 & 47 & 34.9 & 35.0 \\
\hline$\# 5$ & DC - Y. ent. & 2.73 & 172 & 0.59 & 1.33 & 172 & 48 & 35.0 & 34.7 \\
\hline \#6 & DC - Y. ent. & 2.73 & 134 & 0.73 & 1.04 & 226 & 48 & 35.0 & 34.7 \\
\hline \#7 & DC - Y. ent. & 2.73 & 161 & 0.86 & 2.18 & 170 & 49 & 35.0 & 34.5 \\
\hline$\# 8$ & DC - Y. ent. & 2.73 & 81 & 1.18 & 1.01 & 52 & 49 & 35.0 & 34.8 \\
\hline$\# 9 *$ & DC - Y. ent. & 2.73 & 185 & 0.71 & 1.97 & 109 & 49 & 35.0 & 34.0 \\
\hline$\# 10^{*}$ & J774 - E. coli & 2.73 & 167 & 0.73 & 2.40 & 75 & 43 & 35.0 & 32.6 \\
\hline \#11 & DC - M. mar. & 4.24 & 198 & 0.70 & 2.82 & 89 & 40 & 48.9 & 43.7 \\
\hline \#12 & $D C-M$. mar. & 4.24 & 142 & 0.86 & 2.02 & 77 & 42 & 50.0 & 50.0 \\
\hline$\# 13^{*}$ & DC - M. mar. & 4.24 & 165 & 0.92 & 3.06 & 68 & 35 & 47.3 & 48.3 \\
\hline$\# 14$ & DC - M. mar. & 4.24 & 117 & 0.77 & 1.73 & 151 & 40 & 49.6 & 49.1 \\
\hline \#15 & $D C-M$. mar. & 4.24 & 331 & 0.89 & 3.99 & 24 & 32 & 51.0 & 39.4 \\
\hline \#16 & DC - M. mar. & 4.24 & 353 & 1.09 & 4.50 & 51 & 33 & 48.1 & 43.8 \\
\hline
\end{tabular}

In order to get 5-20 nm nanogold fiducials into eukaryotic cells, we incubated the cells with BSA-gold for $1 \mathrm{~h}$ before vitrification. Some cytotoxicity has been observed after longer incubation times depending on cell type concentration, incubation time, and gold particle size (Khlebtsov and Dykman, 2011; Leopold et al., 2017; Lillo et al., 2020). We successfully used this method to get BSA-gold fiducials into FIBlamellae of different cell types, including dendritic cells (Fig. 1C, Movie S2), primary human blood monocytes and dendritic cells infected with Yersinia enterocolitica (Fig. 1D, Movie S3), and the mouse macrophage cell line J774 infected with Escherichia coli (Fig. 1E, Movie S4); we observed BSA-gold in different endosomal organelles including endosomes, lysosomes, phagosomes as well as autophagosomal organelles after fusion with endosomal organelles. We therefore recommend to use incubation times up to $2 \mathrm{~h}$, which is sufficient for a good distribution of BSA-gold into endosomal organelles.

To quantify the distribution of BSA-gold within the cell, we recorded EM tilescan overviews of epon section (Faas et al., 2012) of J774 cells after $1 \mathrm{~h}$ of BSA gold endocytosis, with a similar thickness to FIB lamellae (Sup. Fig. 1). We found a good distribution of BSA gold in endocytotic organelles throughout the cytosol with at least several thousand $10 \mathrm{~nm}$ BSA-gold particles in sections of different cells. The expected number of BSA-gold particles present in tilt-series depends on many factors, including the cell type, the size of the field of view and the subcellular localization of the biological target of interest. Based on the distribution in Sup. Fig. 1 and our experience in applying this techniques to different biological targets, we estimate the chance of having sufficient gold for alignment of a CET tilt-series recorded on a FIB-lamellae to be between 50 and $100 \%$ for a target that is not localized in the nucleus. The strategy of endocytosis of BSA-gold was particularly successful for a dataset with over 150 CET tilt-series recorded on FIB-lamellae of intracellular $Y$. enterocolitica, where each tilt-series could be aligned with a large number of BSA-gold particles. A wide range of cell types are known to endocytose BSA-gold (Geoffroy and Becker, 1984; Peters et al., 1995; Bright et al., 1997; De Heidi et al., 1999; Lopes da Silva et al., 2012; Galmes et al., 2015), making this method widely applicable to many different samples for biological targets in or near organelles of endosomal origin.

\section{Assessment of fiducial-based tilt-series alignment}

The use of this method allows for BSA-gold to be used as fiducials to align tilt-series recorded on cryo-FIB lamellae of eukaryotic cells, which has several advantages over cross-correlation and feature-based, fiducial-less alignment. BSA-gold fiducials are very electron dense compared to the surrounding cellular material, which results in a high contrast and accurate tracking of the center of the BSA-gold particles, even at high tilt-angles where the electrons have a longer path through the biological sample. Because the BSA-gold particles are mostly spherical, they look nearly the same from all tilt angles, which makes it more accurate to determine the center. Accurate tracking of a large number of fiducials distributed throughout the sample in all dimensions allows a more precise determination of the tilt angles, rotations, and magnification as well as local correction of tilt-series and correction for motion of the sample during acquisition of the tilt-series (Mastronarde and Held, 2017; Fernandez et al., 2018, 2019).

To compare the alignment quality of CET tilt-series recorded on FIBlamellae aligned with BSA-gold or fiducial-less patch-tracking, we independently aligned sixteen tilt-series with both methods (Fig. 2, Table 1). Alignment with BSA-gold results in fewer reconstruction artifacts in both BSA-gold fiducials (Fig. 2B, C) and biological structures (Fig. 2D, E). When comparing the alignment parameters, we see that alignment with BSA-gold resulted in lower residual errors and that more contours could be tracked at all tilt-images (Table 1). These results indicate that alignment of tilt-series recorded on FIB-lamella with BSAgold as fiducials gives higher quality tomographic reconstructions compared to fiducial-less patch tracking. Improvements in tilt-series alignment are known to be able to improve the resolution obtained with subtomogram averaging (Fernandez et al., 2018, 2019; Himes and Zhang, 2018; Chen et al., 2019). We therefore expect that the improved tilt-series alignment observed with BSA-gold may improve the resolution obtained with subtomogram averaging.

\section{Conclusion}

In this technical note, we provide a simple method to get BSA-gold fiducials into eukaryotic cells via endocytosis, which enables the 
accurate alignment of CET tilt-series recorded on FIB-lamellae with gold fiducials. Furthermore, we demonstrate its successful application for different cell types and show its subcellular distribution. Whether sufficient BSA-gold is consistently present around a biological target of interest for tilt-series alignment depends on different factors. Thus, the suitability of this technique will need to be assessed for different use cases.

We show better tilt-series alignment with BSA-gold compared to fiducial-less alignment methods that are commonly employed. The strong contrast of the BSA-gold also benefits fiducial-less alignment, so larger benefits are expected when comparing FIB-lamellae with and without BSA-gold. This method supports the use of CET on FIB-lamellae to study the structure of protein complexes in their native cellular environment. One of the downsides of the use of gold fiducials is that they may cause streak artifacts near the gold particles caused by the missing wedge. This can be mostly negated by the computational removal of the gold particle in the tilt-series during the reconstruction process.

Recently, the use of per-particle refinement of tilt parameters has shown great promise of improving the resolution of the final subtomogram averages, even on in situ samples (Himes and Zhang, 2018; Chen et al., 2019; O'Reilly et al., 2020). BSA-gold fiducials can be used in conjunction with this technique for high-quality, (semi-) automated alignment of the tilt-series prior to per-particle refinement. Other uses include research questions that do not require subtomogram averaging as well as for traditional electron tomography at room temperature. Additionally, the use of BSA-gold also helps in identifying organelles, since they are only present in organelles of endosomal origin. Finally, endocytosis of fluorescently labeled BSA-gold (Fokkema et al., 2018) may potentially be used as fiducials to accurately correlate cryofluorescence microscopy data with CET on FIB lamellae (Wolff et al., 2016; Bharat et al., 2018; Kuba et al., 2021).

\section{CRediT authorship contribution statement}

Casper Berger: Conceptualization, Investigation, Writing - original draft. Raimond B.G. Ravelli: Writing - review \& editing. Carmen López-Iglesias: Writing - review \& editing. Peter J. Peters: Conceptualization, Writing - review \& editing.

\section{Declaration of Competing Interest}

The authors declare that they have no known competing financial interests or personal relationships that could have appeared to influence the work reported in this paper.

\section{Acknowledgments}

The UM acknowledge co-funding by the PPP Allowance made available by Health $\sim$ Holland, Top Sector Life Sciences \& Health, to stimulate public-private partnerships, under project number LHSM18067, as well as from the Netherlands Organisation for Scientific Research (NWO) in the framework of the National Roadmap NEMI project number 184.034.014.

We thank the Microscopy CORE Lab of M4I-FHML at Maastricht University for their technical and scientific support. We thank Hang Nguyen for editing the manuscript. We thank the Cell Microscopy Core (Department of Cell Biology, University Medical Center Utrecht) for their kind gift of the $5 \mathrm{~nm}$ BSA-gold labeled with Alexa 488. We thank Christof Diebolder and Rebecca Dillard for their assistance with data acquisition with a Titan Krios at the Netherlands Centre for Electron Nanoscopy (NeCEN).

\section{Appendix A. Supplementary data}

Supplementary data to this article can be found online at https://doi. $\operatorname{org} / 10.1016 / j . j s b .2021 .107698$.

\section{References}

Albert, S., Wietrzynski, W., Lee, C.-W., Schaffer, M., Beck, F., Schuller, J.M., Salomé, P. A., Plitzko, J.M., Baumeister, W., Engel, B.D., 2020. Direct visualization of degradation microcompartments at the ER membrane. Proc. Natl. Acad. Sci. 117 (2), 1069-1080. https://doi.org/10.1073/pnas.1905641117.

Bäuerlein, F.J.B., Saha, I., Mishra, A., Kalemanov, M., Martínez-Sánchez, A., Klein, R., Dudanova, I., Hipp, M.S., Hartl, F.U., Baumeister, W., Fernández-Busnadiego, R., 2017. In Situ Architecture and Cellular Interactions of PolyQ Inclusions. Cell 171 (1), 179-187.e10. https://doi.org/10.1016/j.cell.2017.08.009.

Bay, H., Ess, A., Tuytelaars, T., Van Gool, L., 2008. Speeded-Up Robust Features (SURF). Comput. Vis. Image Underst. 110 (3), 346-359. https://doi.org/10.1016/j. cviu.2007.09.014.

Bharat, T.A.M., Hoffmann, P.C., Kukulski, W., 2018. Correlative Microscopy of Vitreous Sections Provides Insights into BAR-Domain Organization In Situ. Structure 26 (6), 879-886.e3. https://doi.org/10.1016/j.str.2018.03.015.

Bolaños, K., Kogan, M.J., Araya, E., 2019. Capping gold nanoparticles with albumin to improve their biomedical properties. Int. J. Nanomedicine 14, 6387-6406. https:// doi.org/10.2147/IJN.S210992.

Bright, N.A., Reaves, B.J., Mullock, B.M., Luzio, J.P., 1997. Dense core lysosomes can fuse with late endosomes and are re-formed from the resultant hybrid organelles. J. Cell Sci. 110, 2027-2040.

Chen, M., Bell, J.M., Shi, X., Sun, S.Y., Wang, Z., Ludtke, S.J., 2019. A complete data processing workflow for cryo-ET and subtomogram averaging. Nat. Methods 16 (11), 1161-1168. https://doi.org/10.1038/s41592-019-0591-8.

de Wit, H., Lichtenstein, Y., Geuze, H.J., Kelly, R.B., van der Sluijs, P., Klumperman, J., Pfeffer, S.R., 1999. Synaptic vesicles form by budding from tubular extensions of sorting endosomes in PC12 cells. Mol. Biol. Cell 10 (12), 4163-4176. https://doi. org/10.1091/mbc.10.12.4163.

Dobro, M.J., Oikonomou, C.M., Piper, A., Cohen, J., Guo, K., Jensen, T., Tadayon, J., Donermeyer, J., Park, Y., Solis, B.A., Kjær, A., Jewett, A.I., McDowall, A.W., Chen, S., Chang, Y.-W., Shi, J., Subramanian, P., Iancu, C.V., Li, Z., Briegel, A., Tocheva, E.I., Pilhofer, M., Jensen, G.J., Silhavy, T.J., 2017. Uncharacterized Bacterial Structures Revealed by Electron Cryotomography. J. Bacteriol. 199 (17) https://doi.org/ 10.1128/JB.00100-17.

Faas, F.G.A., Avramut, M.C., M. van den Berg, B., Mommaas, A.M., Koster, A.J., Ravelli, R.B.G., 2012. Virtual nanoscopy: Generation of ultra-large high resolution electron microscopy maps. J. Cell Biol 198 (3), 457-469. https://doi.org/10.1083/ jcb.20120114010.1083/jcb.201201140.dv.

Fernandez, J.-J., Li, S., Agard, D.A., 2019. Consideration of sample motion in cryotomography based on alignment residual interpolation. J. Struct. Biol. 205 (3), 1-6. https://doi.org/10.1016/j.jsb:2019.01.005.

Fernandez, J.-J., Li, S., Bharat, T.A.M., Agard, D.A., 2018. Cryo-tomography tilt-series alignment with consideration of the beam-induced sample motion. J. Struct. Biol. 202 (3), 200-209. https://doi.org/10.1016/j.jsb:2018.02.001.

Fokkema, J., Fermie, J., Liv, N., van den Heuvel, D.J., Konings, T.O.M., Blab, G.A., Meijerink, A., Klumperman, J., Gerritsen, H.C., 2018. Fluorescently Labelled Silica Coated Gold Nanoparticles as Fiducial Markers for Correlative Light and Electron Microscopy. Sci. Rep. 8, 13625. https://doi.org/10.1038/s41598-018-31836-1.

Frederik, P.M., Hubert, D.H.W., 2005. Cryoelectron Microscopy of Liposomes. Methods in Enzymology. 431-448. https://doi.org/10.1016/S0076-6879(05)91024-0.

Galmes, R., ten Brink, C., Oorschot, V., Veenendaal, T., Jonker, C., van der Sluijs, P., Klumperman, J., 2015. Vps33B is required for delivery of endocytosed cargo to lysosomes. Traffic 16 (12), 1288-1305. https://doi.org/10.1111/tra.12334.

Geoffroy, J.S., Becker, R.P., 1984. Endocytosis by endothelial phagocytes: uptake of bovine serum albumin-gold conjugates in bone marrow. J. Ultrasructure Res. 89 (3), 223-239. https://doi.org/10.1016/S0022-5320(84)80039-8.

Gruska, M., Medalia, O., Baumeister, W., Leis, A., 2008. Electron tomography of vitreous sections from cultured mammalian cells. J. Struct. Biol. 161 (3), 384-392. https:// doi.org/10.1016/j.jsb:2007.10.008.

Han, R., Zhang, F.a., Wan, X., Fernández, J.-J., Sun, F., Liu, Z., 2014. A marker-free automatic alignment method based on scale-invariant features. J. Struct. Biol. 186 (1), 167-180. https://doi.org/10.1016/j.jsb:2014.02.011.

Harapin, J., Börmel, M., Sapra, K.T., Brunner, D., Kaech, A., Medalia, O., 2015. Structural analysis of multicellular organisms with cryo-electron tomography. Nat. Methods 12 (7), 634-636. https://doi.org/10.1038/nmeth.3401.

Himes, B.A., Zhang, P., 2018. emClarity: software for high-resolution cryo-electron tomography and subtomogram averaging. Nat. Methods 15 (11), 955-961. https:// doi.org/10.1038/s41592-018-0167-z.

Hoiczyk, E., 2000. Structure and sequence analysis of Yersinia YadA and Moraxella UspAs reveal a novel class of adhesins. EMBO J. 19, 5989-5999. https://doi.org/ 10.1093/emboj/19.22.5989.

Jasnin, M., Beck, F., Ecke, M., Fukuda, Y., Martinez-Sanchez, A., Baumeister, W., Gerisch, G., 2019. The Architecture of Traveling Actin Waves Revealed by CryoElectron Tomography. Structure 27 (8), 1211-1223.e5. https://doi.org/10.1016/j. str.2019.05.009.

Karimi Nejadasl, F., Karuppasamy, M., Newman, E.R., McGeehan, J.E., Ravelli, R.B.G., 2013. Non-rigid image registration to reduce beam-induced blurring of cryo-electron microscopy images. J. Synchrotron Radiat. 20 (1), 58-66. https://doi.org/10.1107/ S0909049512044408.

Khlebtsov, N., Dykman, L., 2011. Biodistribution and toxicity of engineered gold nanoparticles: a review of in vitro and in vivo studies. Chem. Soc. Rev. 40 (3), 1647-1671. https://doi.org/10.1039/C0CS00018C. 
Kleijmeer, M.J., Morkowski, S., Griffith, J.M., Rudensky, A.Y., Geuze, H.J., 1997. Major histocompatibility complex class II compartments in human and mouse B lymphoblasts represent conventional endocytic compartments. J. Cell Biol. 139, 639-649. https://doi.org/10.1083/jcb.139.3.639.

Kuba, Jakub, Mitchels, John, Hovorka, Miloš, Erdmann, Philipp, Berka, Lukáš, Kirmse, Robert, König, Julia, de Bock, Jan, Goetze, Bernhard, Rigort, Alexander, 2021. Advanced cryo-tomography workflow developments - correlative microscopy, milling automation and cryo-lift-out. J. Microsc. 281 (2), 112-124. https://doi.org/ 10.1111/jmi.v281.210.1111/jmi.12939.

Kuhlbrandt, W., 2014. The Resolution Revolution. Science 343 (6178), 1443-1444. https://doi.org/10.1126/science:1251652.

Leopold, L.F., Tódor, I.S., Diaconeasa, Z., Rugină, D., Ştefancu, A., Leopold, N., Coman, C., 2017. Assessment of PEG and BSA-PEG gold nanoparticles cellular interaction. Colloids Surfaces A Physicochem. Eng. Asp. 532, 70-76. https://doi.org/ 10.1016/j.colsurfa.2017.06.061.

Lillo, C.R., Calienni, M.N., Rivas Aiello, B., Prieto, M.J., Rodriguez Sartori, D., Tuninetti, J., Toledo, P., Alonso, S.D.V., Moya, S., Gonzalez, M.C., Montanari, J., Soler-Illia, G.J.A.A., 2020. BSA-capped gold nanoclusters as potential theragnostic for skin diseases: Photoactivation, skin penetration, in vitro, and in vivo toxicity. Mater. Sci. Eng. C 112, 110891. https://doi.org/10.1016/j.msec.2020.110891.

Lopes da Silva, M., Thieleke-Matos, C., Cabrita-Santos, L., Ramalho, J.S., WavreShapton, S.T., Futter, C.E., Barral, D.C., Seabra, M.C., 2012. The Host Endocytic Pathway is Essential for Plasmodium berghei Late Liver Stage Development. Traffic 13 (10), 1351-1363. https://doi.org/10.1111/tra.2012.13.issue-1010.1111/j.16000854.2012.01398.x.

Lowe, D.G., 2004. Distinctive Image Features from Scale-Invariant Keypoints. Int. J. Comput. Vis. 60 (2), 91-110. https://doi.org/10.1023/B: VISI.0000029664.99615.94.

Mahamid, J., Pfeffer, S., Schaffer, M., Villa, E., Danev, R., Kuhn Cuellar, L., Forster, F., Hyman, A.A., Plitzko, J.M., Baumeister, W., 2016. Visualizing the molecular sociology at the HeLa cell nuclear periphery. Science 351 (6276), 969-972. https:// doi.org/10.1126/science:aad8857.

Marko, M., Hsieh, C., Schalek, R., Frank, J., Mannella, C., 2007. Focused-ion-beam thinning of frozen-hydrated biological specimens for cryo-electron microscopy. Nat. Methods 4 (3), 215-217. https://doi.org/10.1038/nmeth1014.

Mastronarde, D.N., Held, S.R., 2017. Automated tilt series alignment and tomographic reconstruction in IMOD. J. Struct. Biol. 197 (2), 102-113. https://doi.org/10.1016 j.jsb:2016.07.011.

Noble, A.J., Stagg, S.M., 2015. Automated batch fiducial-less tilt-series alignment in Appion using Protomo. J. Struct. Biol. 192 (2), 270-278. https://doi.org/10.1016/j. jsb:2015.10.003.

O'Reilly, F.J., Xue, L., Graziadei, A., Sinn, L., Lenz, S., Tegunov, D., Blötz, C., Singh, N., Hagen, W.J.H., Cramer, P., Stülke, J., Mahamid, J., Rappsilber, J., 2020. In-cell architecture of an actively transcribing-translating expressome. Science 369 (6503), 554-557. https://doi.org/10.1126/science:abb3758.

Owen, C.H., Landis, W.J., 1996. Alignment of electron tomographic series by correlation without the use of gold particles. Ultramicroscopy 63 (1), 27-38. https://doi.org/ 10.1016/0304-3991(95)00154-9.
Peters, P.J., Raposo, G., Neefjes, J.J., Oorschot, V., Leijendekker, R.L., Geuze, H.J., Ploegh, H.L., 1995. Major histocompatibility complex class II compartments in human B lymphoblastoid cells are distinct from early endosomes. J. Exp. Med. 182, 325-334. https://doi.org/10.1084/jem.182.2.325.

Pierson, J., Ziese, U., Sani, M., Peters, P.J., 2011. Exploring vitreous cryo-section-induced compression at the macromolecular level using electron cryo-tomography; $80 \mathrm{~S}$ yeast ribosomes appear unaffected. J. Struct. Biol. 173 (2), 345-349. https://doi.org/ 10.1016/j.jsb:2010.09.017.

Ravelli, R.B.G., Nijpels, F.J.T., Henderikx, R.J.M., Weissenberger, G., Thewessem, S, Gijsbers, A., Beulen, B.W.A.M.M., López-Iglesias, C., Peters, P.J., 2020. Cryo-EM structures from sub-nl volumes using pin-printing and jet vitrification. Nat. Commun. 11, 2563. https://doi.org/10.1038/s41467-020-16392-5.

Russo, C.J., Passmore, L.A., 2014. Ultrastable gold substrates for electron cryomicroscopy. Science 346 (6215), 1377-1380. https://doi.org/10.1126/science: 1259530.

Schaffer, M., Mahamid, J., Engel, B.D., Laugks, T., Baumeister, W., Plitzko, J.M., 2017. Optimized cryo-focused ion beam sample preparation aimed at in situ structural studies of membrane proteins. J. Struct. Biol. 197 (2), 73-82. https://doi.org/ 10.1016/j.jsb:2016.07.010.

Schindelin, J., Arganda-Carreras, I., Frise, E., Kaynig, V., Longair, M., Pietzsch, T., Preibisch, S., Rueden, C., Saalfeld, S., Schmid, B., Tinevez, J.-Y., White, D.J., Hartenstein, V., Eliceiri, K., Tomancak, P., Cardona, A., 2012. Fiji: an open-source platform for biological-image analysis. Nat. Methods 9 (7), 676-682. https://doi. org/10.1038/nmeth.2019.

Turoňová, B., Schur, F.K.M., Wan, W., Briggs, J.A.G., 2017. Efficient 3D-CTF correction for cryo-electron tomography using NovaCTF improves subtomogram averaging resolution to 3.4 Å. J. Struct. Biol. 199 (3), 187-195. https://doi.org/10.1016/j.jsb: 2017.07.007.

Weiss, G.L., Kieninger, A.-K., Maldener, I., Forchhammer, K., Pilhofer, M., 2019. Structure and Function of a Bacterial Gap Junction Analog. Cell 178 (2), 374-384. e15. https://doi.org/10.1016/j.cell.2019.05.055.

Wolff, G., Hagen, C., Grünewald, K., Kaufmann, R., 2016. Towards correlative superresolution fluorescence and electron cryo-microscopy. Biol. Cell 108 (9), 245-258. https://doi.org/10.1111/boc.v108.910.1111/boc.201600008.

Wolff, G., Limpens, R.W.A.L., Zevenhoven-Dobbe, J.C., Laugks, U., Zheng, S., de Jong, A. W.M., Koning, R.I., Agard, D.A., Grünewald, K., Koster, A.J., Snijder, E.J., Bárcena, M., 2020. A molecular pore spans the double membrane of the coronavirus replication organelle. Science 369 (6509), 1395-1398. https://doi.org/10.1126/ science:abd3629.

Woodward, C.L., Mendonça, L.M., Jensen, G.J., 2015. Direct visualization of vaults within intact cells by electron cryo-tomography. Cell. Mol. Life Sci. 72 (17), 3401-3409. https://doi.org/10.1007/s00018-015-1898-y.

Zheng, S.Q., Palovcak, E., Armache, J.-P., Verba, K.A., Cheng, Y., Agard, D.A., 2017. MotionCor2: anisotropic correction of beam-induced motion for improved cryoelectron microscopy. Nat. Methods 14 (4), 331-332. https://doi.org/10.1038/ nmeth.4193. 